OPEN ACCESS

Edited by:

Alfredo Pulvirenti,

University of Catania, Italy

Reviewed by:

Gregorio Iraola,

Institut Pasteur de Montevideo,

Uruguay

Rifat Hamoudi,

University of Sharjah, United Arab

Emirates

${ }^{*}$ Correspondence:

Peng Cai

cp@mail.hzau.edu.cn

Specialty section:

This article was submitted to

Computational Genomics,

a section of the journal

Frontiers in Genetics

Received: 08 May 2021

Accepted: 06 August 2021

Published: 07 September 2021

Citation:

Gao C-H, Yu G and Cai P (2021)

ggVennDiagram: An Intuitive,

Easy-to-Use, and Highly

Customizable R Package to Generate

Venn Diagram.

Front. Genet. 12:706907.

doi: 10.3389/fgene.2021.706907

\section{ggVennDiagram: An Intuitive, Easy-to-Use, and Highly Customizable R Package to Generate Venn Diagram}

\author{
Chun-Hui Gao', Guangchuang Yu² and Peng Cai ${ }^{1 *}$
}

'State Key Laboratory of Agricultural Microbiology, State Environmental Protection Key Laboratory of Soil Health and Green Remediation, College of Resources and Environment, Huazhong Agricultural University, Wuhan, China, ${ }^{2}$ Department

of Bioinformatics, School of Basic Medical Sciences, Southern Medical University, Guangzhou, China

Venn diagrams are widely used diagrams to show the set relationships in biomedical studies. In this study, we developed ggVennDiagram, an R package that could automatically generate high-quality Venn diagrams with two to seven sets. The ggVennDiagram is built based on ggplot2, and it integrates the advantages of existing packages, such as venn, RVenn, VennDiagram, and sf. Satisfactory results can be obtained with minimal configurations. Furthermore, we designed comprehensive objects to store the entire data of the Venn diagram, which allowed free access to both intersection values and Venn plot sub-elements, such as set label/edge and region label/filling. Therefore, high customization of every Venn plot sub-element can be fulfilled without increasing the cost of learning when the user is familiar with ggplot2 methods. To date, ggVennDiagram has been cited in more than 10 publications, and its source code repository has been starred by more than 140 GitHub users, suggesting a great potential in applications. The package is an open-source software released under the GPL-3 license, and it is freely available through CRAN (https://cran.rproject.org/package=ggVennDiagram).

\section{Keywords: Venn diagram, grammar of graphic, data visualization, $\mathbf{R}$ software, ggplot2}

\section{INTRODUCTION}

A Venn diagram is a widely used diagram that shows the relationships between multiple sets. In biomedical studies, a Venn diagram is frequently used in distinguishing the membership of various types of data, such as compounds, genes, pathways, and species. When the number of sets is less than five, Venn diagrams are probably the most intuitive form of data visualization, superior to heat maps and tables.

In the $\mathrm{R}$ environment, one of the most popular platforms in biomedical data visualizations, many packages are available to plot a Venn diagram including VennDiagram (Chen and Boutros, 2011), colorfulVennPlot (Noma and Manvae, 2013), venn (Dusa, 2020), nVennR (Quesada, 2021), eulerr (Larsson, 2020), venneuler (Wilkinson, 2011), RVenn (Akyol, 2019), and gplots 
(Warnes et al., 2020), to name a few (see Table $\mathbf{1}$ for a feature comparison of these packages). As one of the most popular software, VennDiagram supports multiple input formats, and it can also generate Euler diagrams in addition to Venn. In addition, venn supports the drawing of Venn diagrams with up to seven sets. RVenn has been developed as a systematic and easy-to-use method for calculating intersecting and overlapping members in Venn diagrams. It is impossible to develop a stateof-the-art Venn tool without absorbing the strengths of the above-mentioned tools.

However, the above-mentioned software packages also have their disadvantages. First of all, these packages have limitations in displaying the difference between various regions in a Venn diagram in spite of the capability of exhibiting the original sets. ColorfulVennPlot and venn do support region filling, but users need to manually specify colors for every region, making it too complicated to be used by ordinary users. Besides, most of these packages lack full support for grammar of graphics, resulting in the failure of adequate integration into the popular ggplot2 ecosystem. In addition, the inputs of some packages are very obscure; thus, it is time-consuming to obtain a qualified input data.

Considering this, we developed ggVennDiagram, an intuitive, easy-to-use, and customizable $\mathrm{R}$ package to generate Venn diagrams, which supports a two- to seven-set Venn plot and generates publication-quality figure with minimal input. Furthermore, we also developed a comprehensive Venn data structure to simplify the expansion of Venn diagrams and make the new presentation of the diagram easy in the future.

\section{RESULTS AND DISCUSSION}

\section{Workflow of ggVennDiagram}

The main function "ggVennDiagram()" accepts a list input and outputs a ggplot object. By measuring the length of input list, it automatically applies internal functions to build a plot in two steps: data pre-processing and visualization. The second step relies on ggplot2's functions; therefore, we mainly focus on explaining the first step as follows.

Data pre-processing then can be divided into two procedures: shape generation, which defines the edges of Venn sets and regions and region value calculation which calculates the region items and performs necessary statistics, such as counting and calculating percentages.

Since the returned data after data pre-processing are compatible with the $s f$ object, these data are directly passed into "geom_sf()"/“geom_sf_label()"/"geom_sf_text()" functions intrinsically provided by ggplot2. Filling colors are mapped to the counts of region items, and a color bar legend is generated automatically to show the difference between different regions (Figure 1A).

\section{Shape Generation}

In ggVennDiagram, we treated all the edges, labels, and polygons as simple features, which refer to a standard to describe how the objects in the real world can be presented in computers, with emphasis on the spatial geometry of these objects. A total of 15 types of simple features are implemented in $\mathrm{R}$, three of which are used to describe all the components of a Venn diagram.

Firstly, the edges of sets are inherited from LINESTRING, which is a sequence of points connected by straight non-selfintersecting lines. Secondly, all the possible intersecting regions are inherited from POLYGON, which is formed by a sequence of closed points. Thirdly, the labels of sets are inherited from POINT, which is a single point used to anchor a short text. Simple features are to define the coordinates of Venn plot components. It is the first time for simple features to be employed in a Venn diagram. Such a design enhances the ability to describe Venn diagram components, making it possible to calculate intersection and overlapping regions between different sets.

To simplify the calculation of simple features, we introduce an S4 class Polygon object which expands the S4 class Venn object derived from RVenn. As those methods are implemented in RVenn, set operation methods are implemented for Polygon object, resulting in the unified set operation functions for the set object Venn and the shape object Polygon.

The shape used in the Venn diagram with less than four sets can be a simple structure, such as a circle or an ellipse, but when the Venn diagram has more than four sets, irregular polygons are required. It is hard to generate irregular polygons with simple geometric functions. Therefore, ggVennDiagram is designed to bear a built-in preprocessed shape data set imported from venn, VennDiagram, and some online materials, which undoubtedly increases the efficiency of shape generation on the user side.

\section{Region Value Calculation}

Region value calculation depends on the RVenn package and new functions written on its defined Venn object. There are a total of $2^{\mathrm{n}}-1$ regions in a Venn diagram, in which $\mathrm{n}$ indicates the number of sets. The member and its number in each region are stored with region IDs in a tibble and joined with the region shape object through unique IDs. Likewise, the member and its number in a set are assigned to the SetEdge through unique IDs in parallel. By doing this, a complete VennPlotData object is generated for subsequent plotting (Figure1B).

\section{Stepwise Self-Customization of Venn Diagrams}

After data pre-processing, ggVennDiagram calls native ggplot2 functions to draw Venn diagrams in four layers (Figures 2A,B). The first layer is to show the number of members in each region, with gradient color filling exhibiting the differences in member number among various regions. The second layer is to show set edges. When an irregular polygon rather than an ellipse and circle is used to draw a Venn diagram, set edges are essential for distinguishing the boundary between different sets. The third layer is to display set labels, and the fourth layer is to exhibit region labels. The data pre-processing function is accessible to users. Thus, it is easy for those familiar with the ggplot2 syntax to revise the details of the image including 
TABLE 1 | Feature comparisons of currently available Venn plot tools (R packages and web tools).

\begin{tabular}{|c|c|c|c|c|c|c|c|c|c|}
\hline & \multirow{2}{*}{$\begin{array}{l}\text { Grammar of } \\
\text { graphics }\end{array}$} & \multicolumn{3}{|c|}{ Data processing } & \multicolumn{3}{|c|}{ Visualization } & \multirow[b]{2}{*}{$\begin{array}{l}\text { Element control (set and } \\
\text { region) }\end{array}$} & \multirow[t]{2}{*}{ References } \\
\hline & & $\begin{array}{l}\text { Access to region } \\
\text { members }\end{array}$ & Input format & $\begin{array}{c}\text { Structured } \\
\text { data storage }\end{array}$ & Region filling ${ }^{a}$ & Shapes & No. of sets & & \\
\hline \multicolumn{10}{|l|}{$\mathrm{R}$ packages } \\
\hline ggVennDiagram & Fully support & Yes & List & Yes & Yes & $\begin{array}{l}\text { Circle, ellipse, } \\
\text { and others }\end{array}$ & $2-7$ & $\begin{array}{l}\text { Set edge/label, region } \\
\text { filling/label }\end{array}$ & Gao, 2021 \\
\hline VennDiagram & No & Yes & List & No & No & Circle, ellipse & $2-5$ & $\begin{array}{l}\text { Set edge/label/filling/area, } \\
\text { region label }\end{array}$ & $\begin{array}{l}\text { Chen and Boutros, } \\
2011\end{array}$ \\
\hline colorfulVennPlot & No & No & Named vector & No & Yes & Circle, ellipse & $2-4$ & Set label, region filling/label & $\begin{array}{l}\text { Noma and Manvae, } \\
2013\end{array}$ \\
\hline venn & No ${ }^{b}$ & No & $\begin{array}{c}\text { List, formula, set } \\
\text { number, Boolean } \\
\text { values }\end{array}$ & No & Yes & $\begin{array}{l}\text { Circle, ellipse, } \\
\text { and others }\end{array}$ & $2-7^{c}$ & $\begin{array}{l}\text { Set edge/label, region } \\
\text { filling/label }\end{array}$ & Dusa, 2020 \\
\hline nVennR & Partial & Yes & List & Yes & No & $\begin{array}{l}\text { Irregular } \\
\text { polygon } \\
\text { (calculated) }\end{array}$ & 2-many & $\begin{array}{l}\text { Set edge/filling/area, region } \\
\text { label }\end{array}$ & Quesada, 2021 \\
\hline eulerr & No & No & $\begin{array}{l}\text { List, data frame, } \\
\text { table, matrix, } \\
\text { named vector }\end{array}$ & No & No & Circle, ellipse & $\begin{array}{l}\text { 2-4, maybe } \\
\text { many }^{d}\end{array}$ & $\begin{array}{l}\text { Set label/filling/area, region } \\
\text { label }\end{array}$ & Larsson, 2020 \\
\hline venneuler & No & No & $\begin{array}{l}\text { Formula, matrix, } \\
\text { character vector }\end{array}$ & No & No & Circle & $\begin{array}{l}2-4, \text { maybe } \\
\text { many }^{d}\end{array}$ & Set label/filling/area & Wilkinson, 2011 \\
\hline RVenn & No & Yes $^{e}$ & $\begin{array}{c}\text { Venn object } \\
\text { (derived from list) }\end{array}$ & No & No & Circle & $2-3$ & Set filling/edge & Akyol, 2019 \\
\hline gplots & No & Yes & List, data frame & No & No & Circle, ellipse & $2-5$ & Set label, region label & Warnes et al., 2020 \\
\hline \multicolumn{10}{|l|}{ Online webtool } \\
\hline InteractiVenn & na & Yes & List (web interface) & na & No & $\begin{array}{l}\text { Circle, ellipse, } \\
\text { and Edwards }\end{array}$ & $2-6$ & Set label/filling, region label & Heberle et al., 2015 \\
\hline Venny & na & Yes & List (web interface) & na & Yes & Circle, ellipse & $2-4$ & Set label, region label/filling & Oliveros, 2007 \\
\hline
\end{tabular}

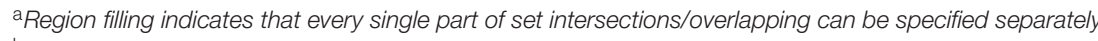

bvenn has a parameter ("ggplot") to enable the output of a ggplot object in plotting.

${ }^{c}$ The five- to seven-set Venn diagram is plotted by ggVennDiagram on the basis of venn.

${ }^{\mathrm{d}}$ When the relationship of different sets is simple enough, eulerr and venneuler can produce an area-proportional Euler plot with more than four sets.

e Set operation of RVenn is expanded in ggVennDiagram to calculate the shapes in different regions. na, not applicable. 


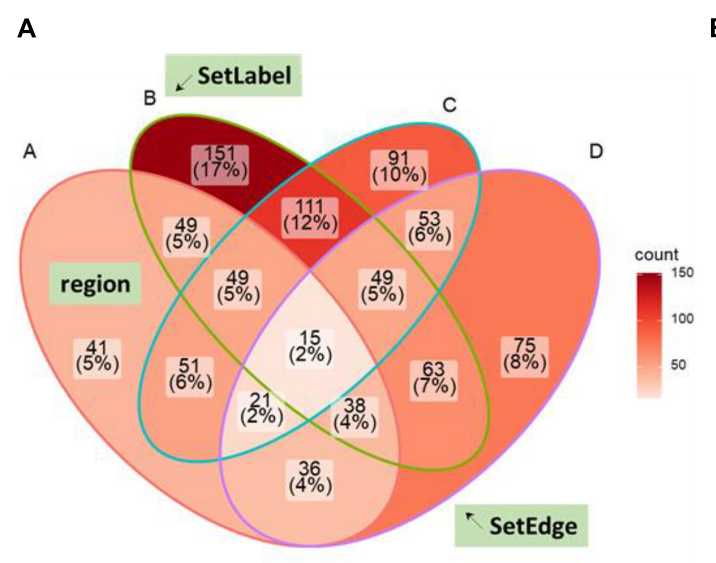

B

Logic structure of ggVennDiagram

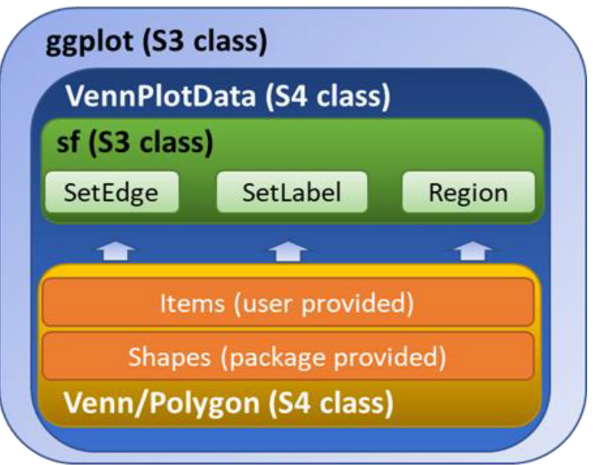

FIGURE 1 | Design of ggVennDiagram. (A) Components of a Venn diagram. SetEdge, SetLabel, and region are highlighted with a green textbox. (B) Logic structure of ggVennDiagram. The Venn diagram plotted by this package is a ggplot object that stores VennPlotData object. The VennPlotData object is further compiled by the simple features described in sf and the Venn/Polygon object introduced from RVenn.

A

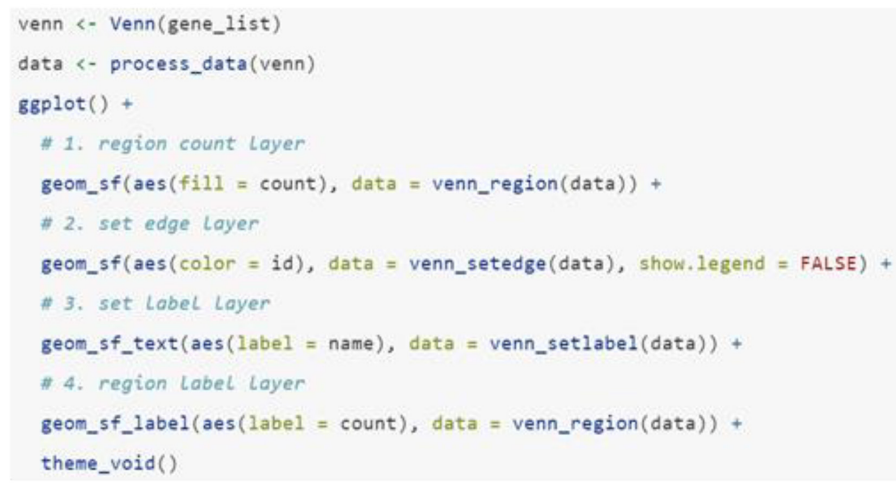

C

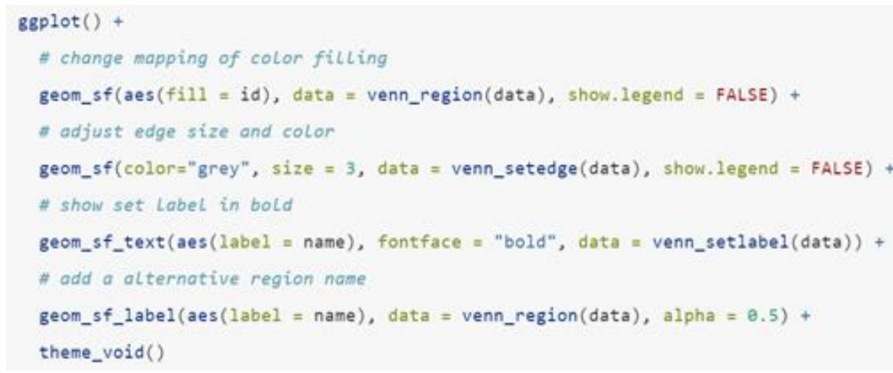

B

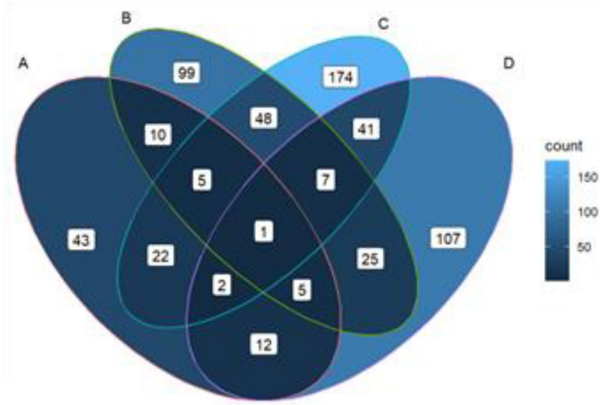

D

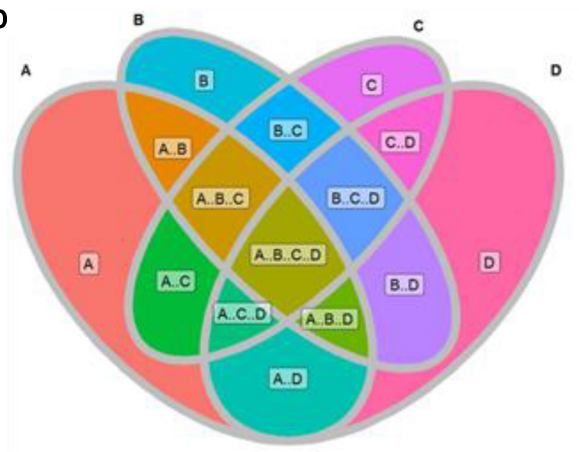

FIGURE 2 | Plotting method of ggVennDiagram. The default manner (A) and returned plot (B) when user calls "ggVennDiagram0" with a four-set list of genes ("gene_list"). (C,D) Stepwise self-customization of the Venn plot by using ordinary ggplot2 functions.

the region fill color, line color/thickness, text style, and so on (Figures 2C,D).

\section{Novel Shapes in Venn Diagrams}

As has been noted above, a set of built-in shapes from ggVennDiagram is used to plot the Venn diagram. By default, only the most appropriate shape is used when the main function
"ggVennDiagram()" is called. However, other applicable shapes can be specified in a stepwise plot, which has been described in the previous section (Figure 3A). In addition, ggVennDiagram provides a series of functions to help users with a novel shape when they know shape coordinates. For example, a six-set Venn diagram can be made up of only six triangles (Figure 3B). To this end, we just need to pass the vertex coordinates and set label 


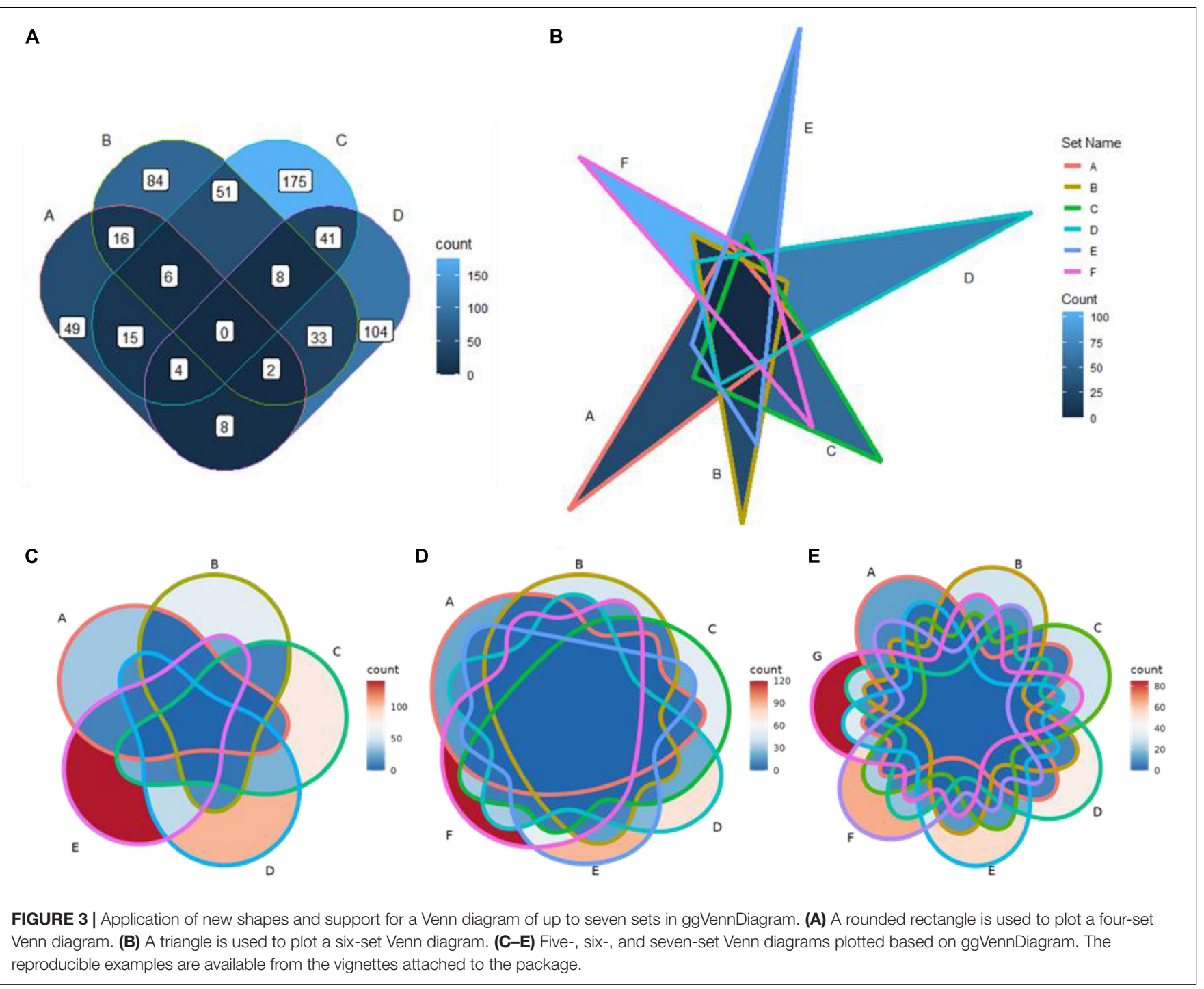

coordinates to the "triangle()" function and "label_position()" function, respectively, and then construct a VennPlotData object with the constructor function "VennPlotData()" (Figure 1B). The generated VennPlotData object now can join with set and calculated region values through "plotData_add_venn()" function, and the resultant data can be used in stepwise customization of the Venn diagram (Figure 3B).

\section{Venn Diagram With More Than Four Sets}

From version 1.0, ggVennDiagram supports Venn diagrams with up to seven sets (Figures 3C-E). This feature is dependent on the shapes imported from another R package venn (Dusa, 2020). However, we insist that Venn diagrams with more than four sets may not be a good choice to display their relationships.

To date, there are three major methods to display set relationships: Venn diagram, Euler diagram, and UpSet plot (Conway et al., 2017). The UpSet plot is a state-of-the-art visualization technique for the quantitative analysis of sets (Lex et al., 2014), and it supports an unlimited number of sets.
When the number of sets is very large, it is more justified to choose the UpSet plot.

\section{Integration of ggVennDiagram Into Bioinformatics Analysis Pipelines}

The first version of ggVennDiagram was released on October 9th, 2019 (version 0.3). Since then, it has been applied to many biomedical research fields. For example, Cook et al. (2020) used ggVennDiagram to show overlapping differentially expressed genes across three sample times (days 1, 3, and 5 ) in both the root and the shoot of canola. Besides, Harris et al. (2020) used ggVennDiagram to display that $22.5 \%$ of differentially expressed genes were shared by treated mice and human patients. Furthermore, Maguire et al. (2020) used ggVennDiagram to confirm that their novel method has low bias and is more sensitive than three other methods for small RNA library preparation. In addition, ggVennDiagram is also used for analyzing the differences between several spatially varied oral metabolomics samples (Ciurli et al., 2021) and for comparing 
A

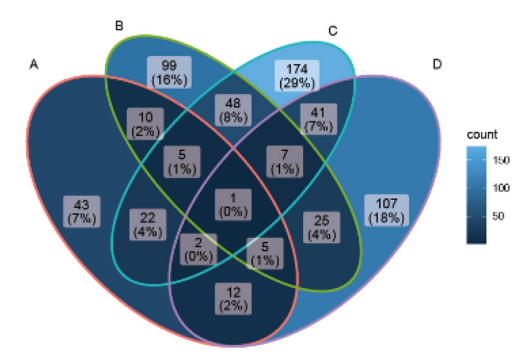

D

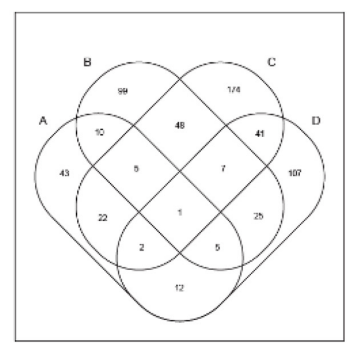

G

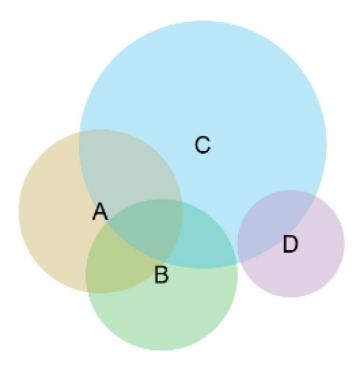

J

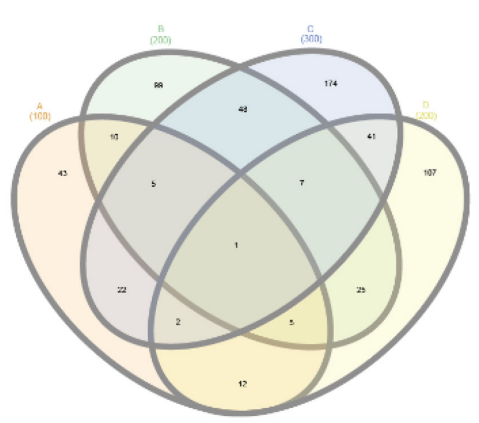

B

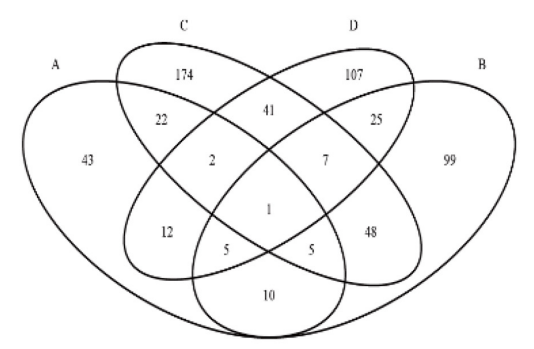

E

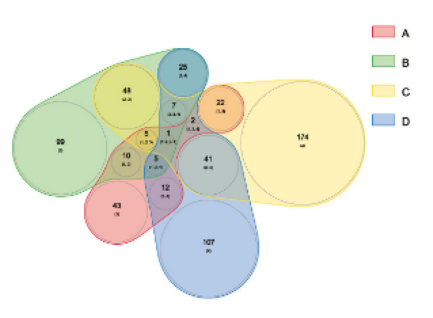

H

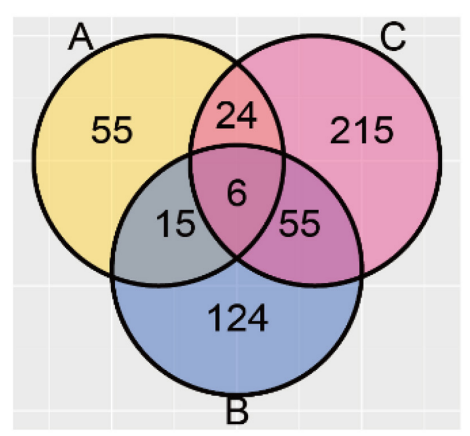

K

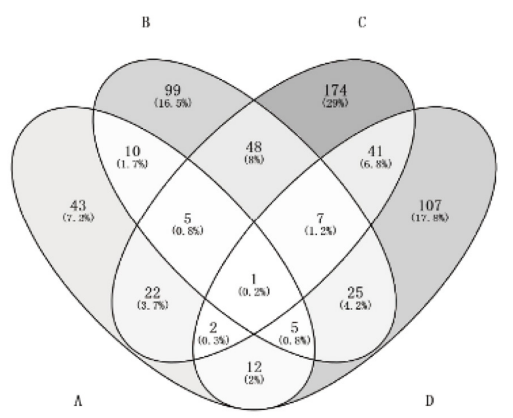

C

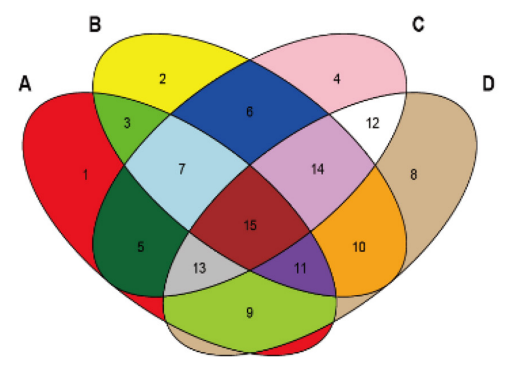

$\mathbf{F}$

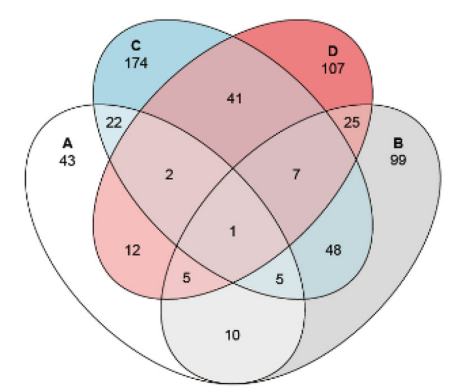

I

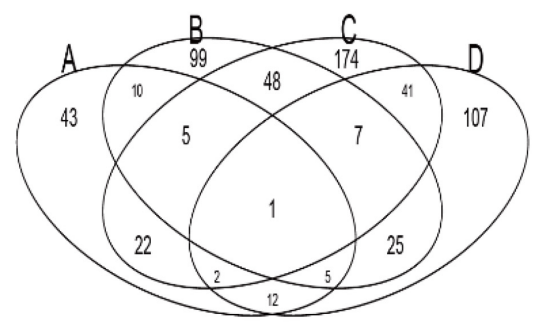

FIGURE 4 | Plots generated by the tools listed in Table 1. The used tools are ggVennDiagram, VennDiagram, colorfulVennPlot, venn, nVennR, eulerr, RVenn, gplots, interactiVenn, and Venny (A-K), respectively. Except for venn (D), venneuler (G), and RVenn (H), all plots are generated with minimal configuration (using default parameters) and the same input data, which is a simulated four-set gene list. The input for venn is a named vector, while the input for venneuler is a qualified expression. Since RVenn do not support four-set Venn plots, only three sets of the gene list are used $\mathbf{( H )}$.

single-nucleotide variants between tumor and non-tumor tissues (Horny et al., 2021). So far, ggVennDiagram has been cited in more than 20 peer-reviewed articles and open-access preprints, as retrieved by Google Scholar. It could be speculated that ggVennDiagram has a very wide range of application scenarios in biomedical studies. 


\section{Feature Comparisons of Currently Available Venn Plot Tools}

Table 1 presents the features of currently available Venn plot tools (see also Figure 4 for the comparison of the generated plots by these tools). First of all, the support for grammar of graphics by nine R packages and two web tools was assessed. Grammar of graphics is a general scheme for data visualization, which breaks up graphs into semantic components, such as scales and layers. Except ggVennDiagram, none of these tools fully support this feature in plotting Venn diagrams.

Additionally, ggVennDiagram takes the lead in the following three aspects of data processing capacity. (1) We can get access to region members by querying the VennPlotData object. (2) It should be noted that we only implement the input of list (as input format). This design is simple enough to understand and prepare, and it is easy to store set members, which is essential for the calculation of region members. (3) Via the design of a layered object, ggVennDiagram can store plotting data into the VennPlotData object (Figure 1B), thus making it possible to query and reuse the target data.

Furthermore, ggVennDiagram is superior in four aspects of visualization. (1) Region filling allows the user to easily identify the differences between various parts of the Venn diagram, and this is one of the key features of ggVennDiagram. Although several other tools have this feature, only ggVennDiagram is fully automatic since it is driven by ggplot2's aesthetic mapping. (2) The ggVennDiagram has built-in shapes consisting of circles, ellipses, and others. Besides, we also provide functions to help users to import self-defined shapes (Figures 3A,B). (3) The ggVennDiagram supports two- to seven-set Venn diagrams, which is adequate for daily use. (4) Element control in ggVennDiagram can be applied for set edge/label and region filling/label, so that it is convenient to set their color/line type/size, and so on (Figures 1A, 2B,D, 3A-E).

Notably, several tools support both Venn and Euler diagrams. However, an Euler diagram has two shortages: firstly, it is area proportional, but the human eye is less sensitive to area than to color; secondly, it only shows relevant relationships, but sometimes, it is impossible to show all intersection regions merely by using simple geometric shapes, such as circles and ellipses. Therefore, we assume that it is more appropriate to use color filling for displaying the difference between different regions in ordinary biomedical studies.

Overall, ggVennDiagram integrates and optimizes a Venn diagram plotting method, exhibiting multiple advantages in

\section{REFERENCES}

Akyol, T. Y. (2019). RVenn: set Operations for Many Sets. Available online at: https://CRAN.R-project.org/package=RVenn (accessed May 1, 2021).

Chen, H., and Boutros, P. C. (2011). VennDiagram: a package for the generation of highly-customizable Venn and Euler diagrams in R. BMC Bioinform. 12:35. doi: 10.1186/1471-21 \05-12-35

Ciurli, A., Liebl, M., Derks, Rico. J. E, Neefjes, J. J. C., and Giera, M. (2021). Spatially resolved sampling for untargeted metabolomics: a new tool for salivomics. iScience 24:102768. doi: 10.1016/j.isci.2021.102768 performance over current existing tools. Compared with webtool, $\mathrm{R}$ scripts are easier to integrate into the existing bioinformatics analysis pipelines to realize automation and batch drawing of Venn diagrams. Therefore, it is necessary and useful to develop ggVennDiagram.

\section{DATA AVAILABILITY STATEMENT}

The ggVennDiagram $\mathrm{R}$ package is open source and freely available on CRAN (https://cran.r-project.org/package= ggVennDiagram) and GitHub (https://github.com/gaospecial/ ggVennDiagram). ggVennDiagram mainly requires $\mathrm{R}(>3.5 .0)$, the ggplot2, and sf packages, and its full function also depends on the plotly package.

\section{AUTHOR CONTRIBUTIONS}

C-HG, GY, and PC wrote this manuscript. C-HG implemented this package with the help of GY. PC supervised the project. All authors contributed to the article and approved the submitted version.

\section{FUNDING}

This work was supported by the National Natural Science Foundation of China (32100090, 41877029, and 41961130383), Royal Society-Newton Advanced Fellowship (NAFR1191017), the National Key Research Program of China (2020YFC1806803), Wuhan Applied Foundational Frontier Project (2019020701011469), and Fundamental Research Funds for the Central Universities (2662021JC012).

\section{ACKNOWLEDGMENTS}

We thank Adrian Duşa for letting us reuse the "venn:::sets" data in his venn package, and this is critical to enable five- to seven-set Venn diagrams in ggVennDiagram. We also thank the GitHub user Yi Liu (@liuyigh) for his contribution on code curation. Great gratitude goes to linguistics Ping Liu from Huazhong Agriculture University, Wuhan, China, for her work on English editing and language polishing.

Conway, J. R., Lex, A., and Gehlenborg, N. (2017). UpSetR: an R package for the visualization of intersecting sets and their properties. Bioinformatics 33, 2938-2940. doi: 10.1093/bioinformatics/btx364

Cook, J., Douglas, G. M., Zhang, J., Glick, B. R., Langille, M. G. I., Liu, K.-H., et al. (2020). Transcriptomic profiling of Brassica napus responses to Pseudomonas aeruginosa. Innate Immun. 27, 143-157. doi: 10.1177/1753425920980512

Dusa, A. (2020). venn: draw venn diagrams. Available online at: https://CRAN.Rproject.org/package=venn (accessed May 1, 2021).

Gao, C.-H. (2021). ggVennDiagram: a ggplot2 implement of venn diagram. Available online at: https://github.com/gaospecial/ggVennDiagram (accessed May 1, 2021). 
Harris, S. E., Poolman, T. M., Arvaniti, A., Cox, R. D., Gathercole, L. L., and Tomlinson, J. W. (2020). The American lifestyle-induced obesity syndrome diet in male and female rodents recapitulates the clinical and transcriptomic features of nonalcoholic fatty liver disease and nonalcoholic steatohepatitis. Am. J. Physiol. Gastrointest. Liver Physiol. 319, G345-G360. doi: 10.1152/ajpgi.00055. 2020

Heberle, H., Meirelles, G. V., da Silva, F. R., Telles, G. P., and Minghim, R. (2015). InteractiVenn: a web-based tool for the analysis of sets through Venn diagrams. BMC Bioinform. 16:169. doi: 10.1186/s12859-01 5-0611-3

Horny, K., Gerhardt, P., Hebel-Cherouny, A., Wülbeck, C., Utikal, J., and Becker, J. C. (2021). Mutational Landscape of Virus- and UV-Associated Merkel Cell Carcinoma Cell Lines Is Comparable to Tumor Tissue. Cancers 13:649. doi: $10.3390 /$ cancers 13040649

Larsson, J. (2020). eulerr: area-proportional Euler and Venn Diagrams With Ellipses. Available online at: https://CRAN.R-project.org/package=eulerr (accessed May 1, 2021).

Lex, A., Gehlenborg, N., Strobelt, H., Vuillemot, R., and Pfister, H. (2014). UpSet: visualization of Intersecting Sets. IEEE Trans. Vis. Comput. Graph. 20, 19831992. doi: 10.1109/TVCG.2014.2346248

Maguire, S., Lohman, G. J. S., and Guan, S. (2020). A low-bias and sensitive small RNA library preparation method using randomized splint ligation. Nucleic Acids Res. 48:e80. doi: 10.1093/nar/gkaa 480

Noma, E., and Manvae, A. (2013). colorfulVennPlot: plot and Add Custom Coloring to Venn Diagrams for 2-Dimensional, 3-Dimensional and 4Dimensional data. Available Online at: https:/CRAN.R-project.org/package= colorfulVennPlot (accessed May 1, 2021).
Oliveros, J. C. (2007). Venny. an Interactive Tool for Comparing Lists with Venn's Diagrams. Available Online at: https://bioinfogp.cnb.csic.es/tools/venny/index. html [Accessed July 6, 2021].

Quesada, V. (2021). nVennR: create n-Dimensional, Quasi-Proportional Venn Diagrams. Available online at: https://CRAN.R-project.org/package $=n V e n n R$ [Accessed March 3, 2021].

Warnes, G. R., Bolker, B., Bonebakker, L., Gentleman, R., Huber, W., Liaw, A., et al. (2020). gplots: various r programming tools for plotting data. Available online at: https://github.com/talgalili/gplots (accessed May 1, 2021).

Wilkinson, L. (2011). venneuler: venn and Euler Diagrams. Available online at: https://CRAN.R-project.org/package=venneuler (accessed May 1, 2021).

Conflict of Interest: The authors declare that the research was conducted in the absence of any commercial or financial relationships that could be construed as a potential conflict of interest.

Publisher's Note: All claims expressed in this article are solely those of the authors and do not necessarily represent those of their affiliated organizations, or those of the publisher, the editors and the reviewers. Any product that may be evaluated in this article, or claim that may be made by its manufacturer, is not guaranteed or endorsed by the publisher.

Copyright (c) 2021 Gao, Yu and Cai. This is an open-access article distributed under the terms of the Creative Commons Attribution License (CC BY). The use, distribution or reproduction in other forums is permitted, provided the original author(s) and the copyright owner(s) are credited and that the original publication in this journal is cited, in accordance with accepted academic practice. No use, distribution or reproduction is permitted which does not comply with these terms. 